\title{
Performance Modeling and Analysis of a Hollow Fiber Membrane System
}

\section{Shuang Liang ${ }^{1}$, Haifeng Zhang ${ }^{2}$, Yubo Zhao' and Lianfa Song ${ }^{3 *}$}

${ }^{1}$ School of Environmental Science and Engineering, Shandong University, Jinan 250100, Shandong, PR China

${ }^{2}$ Department of Chemical Engineering, Northeast Dianli University, Jilin 132012, Jilin, PR China

${ }^{3}$ Department of Civil and Environmental Engineering, Texas Tech University, Lubbock TX 79409-41023, USA

\begin{abstract}
A mathematical model was developed for performance of hollow fiber membrane filtration system by considering both frictional and kinetic pressure losses along the fiber. The model was solved numerically as a whole and analytically with the kinetic term omitted with constant driving pressure as the primary control parameter. Numerical simulations first demonstrated that the kinetic pressure loss in the hollow fiber was negligible compared to the frictional pressure loss for the current hollow fiber membranes. It was further demonstrated that the productivity (exit velocity) of a hollow fiber was greatly affected by the radius of the fiber. The axial velocity reached a plateau value rapidly along a fiber of a small diameter while increased linearly along a fiber of sufficiently large radius. For given membrane materials (resistance) and fiber length, an optimal diameter of the fiber can be determined to maximize exit velocity.
\end{abstract}

Keywords: Hollow fiber membrane; Mathematical modeling; System simulation; Transmembrane pressure; Exit axial velocity; Frictional pressure loss

\section{Nomenclature:}

$$
\begin{aligned}
& d P_{f} \text { differential frictional pressure loss }(\mathrm{Pa}) \\
& d P_{k}: \text { differential kinetic pressure loss }(\mathrm{Pa}) \\
& f: \text { Darcy friction factor } \\
& L: \text { fiber length }(\mathrm{m}) \\
& m \text { : mass in the differential volume in Figure } 1(\mathrm{~m}) \\
& r: \text { inner radius of fiber }(\mathrm{m} / \mathrm{s}) \\
& R_{e}: \text { Reynolds number } \\
& R_{m}: \text { membrane resistance }(1 / \mathrm{m}) \\
& u: \text { average axial lumen velocity }(\mathrm{m} / \mathrm{s}) \\
& u_{0}: \text { exit axial velocity }(\mathrm{m} / \mathrm{s}) \\
& u_{\text {lim }}: \text { limiting exit axial velocity }(\mathrm{m} / \mathrm{s}) \\
& v: \text { permeate flux or velocity }(\mathrm{m} / \mathrm{s}) \\
& v_{0}: \text { permeate velocity or flux at the exit }(\mathrm{m} / \mathrm{s}) \\
& x: \text { axial coordinate }(\mathrm{m})
\end{aligned}
$$

\section{Introduction}

Microfiltration and ultrafiltration membranes in the form of hollow fibers have many desirable advantages over other forms of membranes [1-3], such as high surface/volume ratio, lower cost in fabrication, and diversified applications, etc. In the last couple of decades, hollow fiber membranes have been growingly used in water and wastewater treatments process [4-8], especially in membrane bioreactors (MBRs) for the removal of organic contaminants from wastewater [9-11]. Obviously, there is an urgent need for a better understanding of the performance of hollow fiber membrane system and the affecting factors in order to use this promising technology more effectively and efficiently [12-15].

A hollow fiber with a dead-end operated at either constant transmembrane pressure or constant average flux mode is basically a heterogeneous filtration system, in which the key parameters vary significantly along the fiber length [14-16]. It is well established that the flow pattern and pressure field in a hollow fiber are fully governed by the Navier-Stokes and continuity equations. However, direct solution of Navier-Stokes and continuity equations remains a challenging task $[13,17,18]$ nowadays for the performance modeling and simulation of hollow fiber membrane system. Instead, it would be more practical to develop models that determine the locally varying parameters at constant transmembrane pressure operation mode with the existing theories for tube flows combined with membrane filtration theories. Frictional pressure loss was first considered for the transmembrane pressure variation along the hollow fibers [19-24]. Lately it was realized that the axial flow in the hollow fiber was different from the common tube flow in that the flow accelerates as it flows from the dead-end to the open-end due to the addition of permeate along the fiber. Therefore, the pressure loss as a result of momentum changes might contribute to the change of local transmembrane pressure $[3,25]$. This impact has not been rigorously quantified and assessed in the performance modeling of hollow fiber membrane systems.

When friction of axial flow with the inner fiber wall is the main reason for the variation of local transmembrane pressure, the distribution of transmembrane pressure along the fiber was found to be governed by a second order ordinary differential equation $[2,19]$. An analytical solution of the governing equation was obtained by Chang and Fane [19] and Chang et al. [20,21] with the average initial permeate flux as a control variable. Although the concept of average permeate flux is practically useful in the operation of membrane process, it is unsuitable for exploration of the fundamental principles of the system. One of the

*Corresponding author: Lianfa Song, Department of Civil and Environmenta Engineering, Texas Tech University, Lubbock TX 79409-41023, USA, Tel: 1-806834-4356; Fax: 806-742-3449; E-mail: lianfa.song@ttu.edu

Received January 28, 2016; Accepted February 22, 2016; Published February 24, 2016

Citation: Liang S, Zhang H, Zhao Y, Song L (2016) Performance Modeling and Analysis of a Hollow Fiber Membrane System. J Membra Sci Technol 6: 144 doi:10.4172/2155-9589.1000144

Copyright: ( 2016 Liang S, et al. This is an open-access article distributed under the terms of the Creative Commons Attribution License, which permits unrestricted use, distribution, and reproduction in any medium, provided the original author and source are credited. 
serious drawbacks of the model based on the average permeate flux is that the impact of various parameters on the performance of hollow fiber membrane system cannot be simulated with the model because the average permeate flux that should be used as an indicator of the system performance is already given as the control parameter.

As a matter of fact, the primary control parameter of a membrane process is the transmembrane pressure and the (average) permeate flux should be a natural performance indicator of a membrane system. Even in the membrane processes operated at constant permeate flux operation mode, the constant flux is obtained by adjusting the transmembrane pressure through a feed-back mechanism. Furthermore, the valid range of the average permeate flux for a hollow fiber membrane cannot be determined within the theoretical framework of the model based on average permeate flux. Therefore, there is a chance to pick up a value for the averages permeate flux that is unachievable in the membrane system. On the contrary, when the transmembrane pressure is used as the primary control parameter, the possible maximum permeate flux of a membrane system can be rigorously determined.

This present work presented an effort to address the three fundamental issues mentioned above. First, a mathematical model was developed for a hollow fiber membrane system with the transmembrane pressure as the control parameter. In addition to the frictional pressure loss, the kinetic pressure loss due to fluid acceleration inside of the fiber would be rigorously formulated and assessed. Instead the flow rate or average permeate flux of a fiber membrane, the exit axial flow velocity at the open-end of the fiber was used as the performance indicator of the fiber membrane system. Second, a numerical solution procedure was developed for the complete model with both frictional and kinetic pressure losses and an analytical solution was derived for the reduced model without the kinetic pressure term. The analytical solutions were obtained based on the constant driving pressure operation mode that is different from those based on the average flux as often found in the literature. Third, the performance of hollow fiber system was simulated with the new analytical solution under various conditions. The impacts of fiber length and radius on the performance of hollow fiber membrane system were particularly reported with many interesting findings.

\section{Model Development}

A schematic of a vertical placed hollow fiber is presented in Figure 1a. The end of the fiber on the bottom is sealed (dead-end). Permeate comes out of the open-end of the fiber on the top as a result of either applying a negative suction pressure to the lumen side or a positive pressure from outside dependent on the configuration of the membrane filtration system. In either case, the transmembrane pressure always ascribes a positive value measured from the outside to the inside of the hollow fiber. Because the fiber is always filled with water, the hydraulic head of water depth outside of the fiber has no role to play in the filtration process. The only driving force for permeate is the transmembrane pressure $\Delta \mathrm{P}(x)$. In this sense, the orientation of the hollow fiber in either vertical direction or horizontal direction or any arbitrary direction does not affect the driving force for permeate flux.

In the hollow fiber membrane system, the fibers are commonly characterized with inner radius, fiber length, and membrane resistance. The operating parameter is the transmembrane pressure at the openend of the fiber $\Delta \mathrm{P}$. The overall permeate production rate is a more important concern for hollow fiber membrane system. Therefore, the exit axial velocity at the open-end $\mathrm{u} 0$ is selected over the average membrane flux as the performance indicator of the fiber membrane system. Because these important parameters are defined at the open-

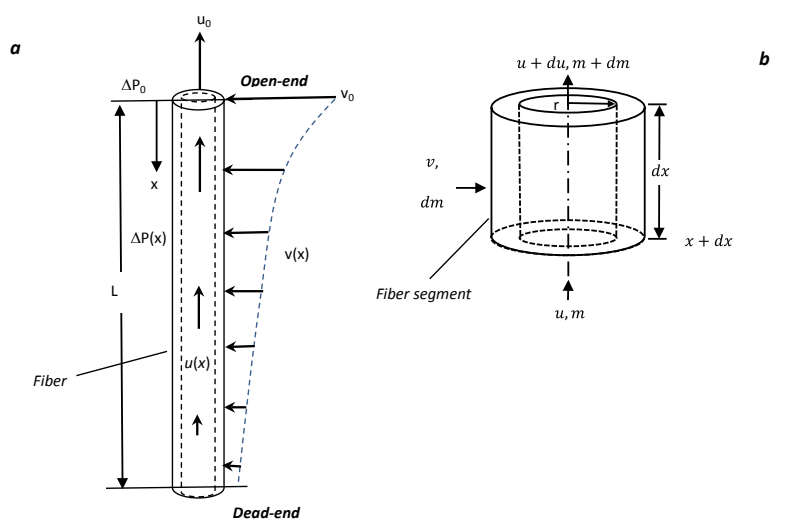

Figure 1: a) Schematic of a hollow fiber with flow pattern and coordinate system. b) Schematic of a segment of hollow fiber and mass and momentum balances.

end of the fiber, it is natural to select the open-end of fiber as the origin of coordinate $\mathrm{x}$. As shown in Figure 1a, the section of the fiber that contributes more to permeate production has smaller $\mathrm{x}$ value in such a coordinate system. A hollow fiber membrane system is completely described by the distributions of transmembrane pressure, permeate flux, and average axial flow velocity.

\section{Average axial velocity and permeate flu}

The average axial velocity inside a hollow fiber is determined by the mass balance relationship with the permeate flux

$$
u(x)=\frac{2}{r} \int_{x}^{L} v(x) d x
$$

where $x$ is the coordinate along the fiber starting from the open-end, $u(x)$ is the average axial velocity with the positive direction defined pointing to the open-end of the fiber, $\mathrm{r}$ is the fiber inner radius, $v(x)$ is the permeate flux, and $L$ is the fiber length. At any location $\mathrm{x}$, the permeate flux of the membrane is calculated with the membrane filtration equation

$$
v(x)=\frac{\Delta P(x)}{\mu R_{m}}
$$

where $\Delta \mathrm{P}(x)$ is the transmembrane pressure, $\mu$ is the viscosity, and $\mathrm{Rm}$ is the membrane resistance. The membrane resistance is a constant along a clean fiber. It needs to point out here that both permeate velocity $v(x)$ and membrane resistance $R m$ is defined with respect to the inner radius of the fiber. In case that the membrane is actually on the outer shell of the fiber, the ratio of outer radius to inner radius is needed to convert these parameters into the right form.

The transmembrane pressure is usually only known at the open-end of a fiber as a given control parameter. The transmembrane pressure decreases along the hollow fiber as a function of location $\mathrm{x}$ and needs to be determined with fluid dynamics. In general, the pressure loss in the hollow fiber can be divided into frictional and kinetic components. The calculations of each component are detailed below separately.

\section{Frictional pressure loss in hollow fiber}

The frictional pressure loss in a small segment of the fiber as shown in Figure $1 b$ is related to the average axial velocity with Darcy-Weisbach equation [26,27].

$$
d P_{f}=f \frac{\rho}{4 r} u^{2} d x
$$


where $d P_{f}$ is the frictional pressure loss, $\mathrm{f}$ is the Darcy friction factor, $\rho$ is the density of water, $\mathrm{dx}$ is the length of the hollow fiber segment. Darcy friction factor of laminar flow in a circular tube is given as $[27,28]$.

$$
f=\frac{64}{R_{e}}=\frac{32 \mu}{\rho u r}
$$

where $R e$ is the Reynolds number, which is defined $R_{-e}=2 \rho$ ur/ $\mu$ for the fiber, and $\mu$ is the viscosity of water.

Substituting Eq. (4) into Eq. (3) and integrating from 0 to $\mathrm{x}$ gives the pressure loss as

$$
\Delta P_{f}(x)=\frac{8 \mu}{r^{2}} \int_{0}^{x} u d x
$$

where $\Delta \mathrm{P}_{f}$ is the frictional pressure loss from the open-end to location $x$.

\section{Kinetic pressure loss in hollow fiber}

Water flow in the fiber lumen accelerates as it moves from the deadend to the open-end because of the addition of permeate along the fiber. Energy is consumed for water movement acceleration. The pressure loss as a result of water acceleration is termed as kinetic pressure loss, which can be calculated from the principle of Newton's second law

$$
\left(\pi r^{2}\right) \mathrm{d} P_{k}=-\frac{d}{d x}\left(\frac{m u^{2}}{2}\right)
$$

where $d P_{k}$ is the pressure loss along the length of $\mathrm{dx}$. The term $\pi \mathrm{r} 2$ is the cross sectional area of the fiber lumen and the term mu2/2 is the kinetic energy carried by the volume of water in the segment of fiber. Using the mass and momentum flows presented in Figure $1 \mathrm{~b}$, it can be found that

$$
\mathrm{d} P_{k}=\frac{2 \rho}{r} u v \mathrm{~d} x
$$

Then the total kinetic pressure loss from the open-end to location $x$ is

$$
\Delta P_{k}(x)=\frac{2 \rho}{r} \int_{0}^{x} u v d x
$$

Therefore the transmembrane pressure at location $x$ of the fiber is determined as

$$
\Delta P(x)=\Delta P-\frac{8 \mu}{r^{2}} \int_{0}^{x} u d x-\frac{2 \rho}{r} \int_{0}^{x} u v d x
$$

where $\Delta \mathrm{P}$ is the transmembrane pressure at the open-end of the hollow fiber membrane.

Up to now, the equations that fully govern the hollow fiber membrane system have been derived, which are Eqs. (1),(2), and (9). These three equations are highly coupled and the analytical solutions are usually difficult to find for the general case. Even for some special cases where analytical solution is available for clean membrane, they may not be valid for fouled membranes because the total hydraulic resistance cannot remain constant for the whole fiber length. Numerical solutions are much more versatile that can be used for both clean and fouled membranes.

\section{Numerical Solution}

In order to construct the numerical solution, the fiber is discretized into $n$ equal segments with the length of $\Delta \mathrm{x}=L / n$ each. The openend of the fiber is designated step as $i=0$, and grids are continuously numbered downward with $i=1,2,3, \ldots$, and $\mathrm{n}$ for the dead-end of the fiber. The axial velocity at step $\mathrm{n}$ is known to be zero because it is the dead-end. In principle, the axial velocity in the previous steps can be determined step by step backward from the step $n$. However, the method has a difficulty to execute because the pressure at the step $n$ is unknown. In this study, an iteration scheme was developed to find the transmembrane pressure for the last step $n$. When this transmembrane pressure is known, the whole problem has been solved at the same time. The method of bisection was used to find the transmembrane pressure for the step $n$. The possible range of transmembrane pressure is initially the $\Delta \mathrm{P}_{\text {max }}$ and $\Delta \mathrm{P}_{\text {min }}$, which are given as

$$
\begin{aligned}
& \Delta P_{\text {max }}=\Delta \mathrm{P} \\
& \Delta P_{\text {min }}=0
\end{aligned}
$$

With the method of bisection, the middle value of the pressure range is assumed the transmembrane pressure, i.e.

$$
\Delta P_{n}=\frac{\Delta P_{\max }+\Delta P_{\min }}{2}
$$

and the permeate flux is calculated as

$$
v_{n}=\frac{\Delta P_{n}}{\mu R_{m}}
$$

For $i<n$, the axial velocity, transmembrane pressure, and permeate flux are determined by

$$
\begin{aligned}
u_{i} & =\frac{2 \Delta x}{r} v_{i+1} \\
\Delta P_{i} & =\Delta P_{i+1}+\frac{8 \mu}{r^{2}} u \Delta x+\frac{2 \rho}{r} u v \Delta x \\
v_{i} & =\frac{\Delta P_{i}}{\mu R_{m}}
\end{aligned}
$$

The process is repeated until $i=0$. During the repetition, whenever $\Delta \mathrm{P}_{i}>\Delta \mathrm{P}$, the upper pressure bound $\Delta \mathrm{P}_{\max }$ is set to the newly determined $\Delta \mathrm{P}_{\mathrm{n}}$ and goes back to Eq. (12) to start over again. By the end of repetition, if $\Delta \mathrm{P}_{0}$ is equal to the driving pressure $\Delta \mathrm{P}$ of the fiber filtration systems, the solution is reached and the process stops. Otherwise, the lower pressure bound $\Delta \mathrm{P}_{\min }$ is set to the newly determined $\Delta \mathrm{P}_{\mathrm{n}}$ and goes back to Eq. (12) to start over again. In each iteration, the domain length of the transmembrane pressure reduces by half. The calculation procedure is presented in the flow diagram shown in Figure 2. The same numerical procedure can be used directly for the fouled fibers where the total hydraulic resistance is a variable instead of a constant.

The numerical solution procedure was coded in $\mathrm{C}++$ language that worked smoothly for all cases of simulations. In most case studies carried out in this study, numerical solutions were obtained in a fraction of second on a common personal computer. The detailed simulation results will be discussed later in the section of Simulations and Discussions. However, one important finding should be pointed out from the numerical solutions: the kinetic pressure loss is less than one percent of the frictional pressure loss for the typical parameters of the current hollow fibers. This finding stimulated the derivation of the analytical solution below.

\section{Analytical Solution}

When the kinetic pressure loss term is omitted from Eq. (9), the remained equation is analytically solvable. Taking derivative of both sides of Eq. (9) without the kinetic pressure loss term results in

$$
\frac{d \Delta P(x)}{d x}=-\frac{8 \mu}{r^{2}} u
$$

Combining Eqs. (1), (2) and (13) gives 


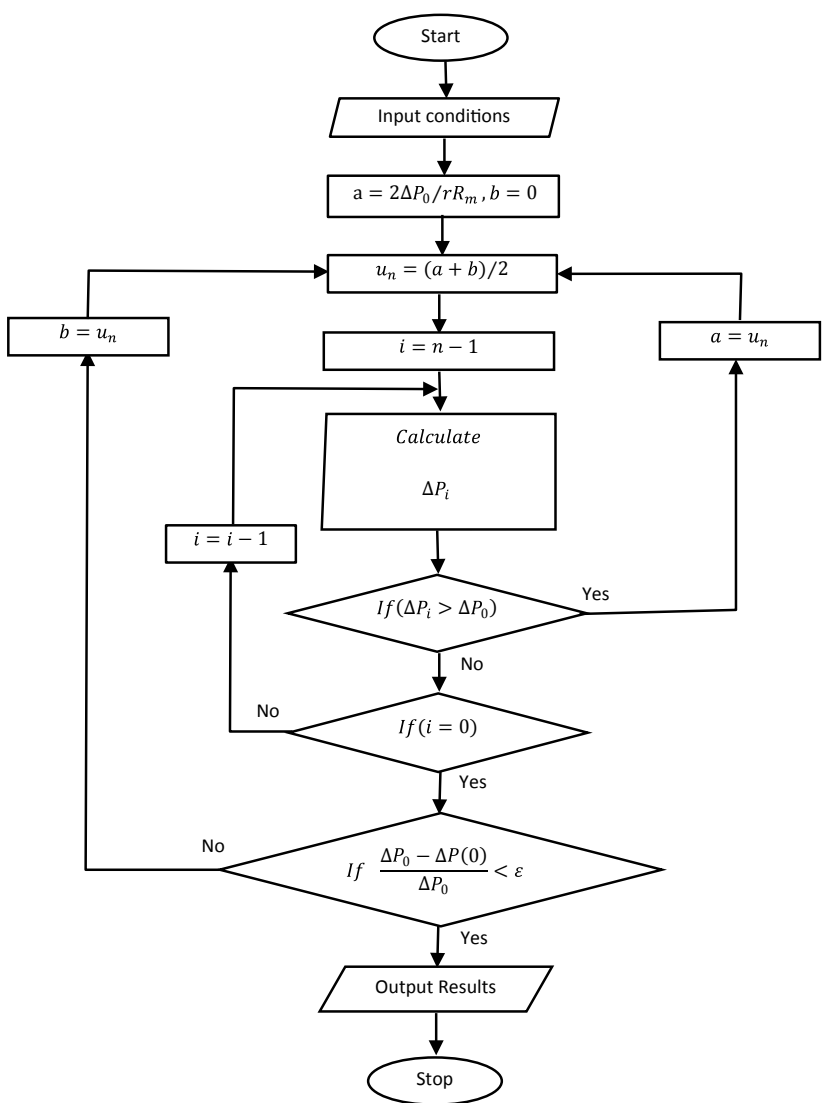

Figure 2: Flowchart for numerical calculation of the performance of hollow fiber filtration system.

$$
\frac{d \Delta P(x)}{d x}=-\frac{16}{r^{3} R_{m}} \int_{x}^{L} \Delta P(x) d x
$$

Taking derivative of both side of Eq. (14) results in

$$
\frac{d^{2} \Delta P(x)}{d x^{2}}=\frac{16}{r^{3} R_{m}} \Delta P(x)
$$

The boundary conditions for the problem under consideration are

$$
\Delta P(0)=\Delta P
$$

and

$$
\left.\frac{d \Delta P(x)}{d x}\right|_{x=L}=0
$$

Eq. (16) means that the pressure at the open-end of the fiber is given as an operational parameter. On the other hand, Eq. (17) reflects the fact that the axial velocity in the hollow fiber is zero at the dead-end.

The solution of Eq. (15) with boundary conditions (16) and (17) is

$$
\Delta P(x)=\Delta P \frac{e^{\lambda(L-x)}+e^{-\lambda(L-x)}}{e^{\lambda L}+e^{-\lambda L}}
$$

where $\lambda$ is given as

$$
\lambda=\frac{4}{\sqrt{r^{3} R_{m}}}
$$

With the known transmembrane pressure distribution, the exit axial velocity of the fiber, which is the performance indicator of the membrane system, is readily determined. Substituting Eq. (1) into Eq. (2) and setting the lower integration limit to 0 , one has the exit axial velocity of the fiber

$$
u_{0}=\frac{2}{r} \int_{0}^{L} \frac{\Delta P(x)}{\mu R_{m}} d x
$$

Substituting Eq. (18) for $\Delta \mathrm{P}(x)$ in Eq. (20), the exit axial velocity is obtained

$$
u_{0}=v_{0} \frac{\sqrt{r R_{m}}}{2} \frac{e^{\lambda L}-e^{-\lambda L}}{e^{\lambda L}+e^{-\lambda L}}=u_{l i m} \frac{e^{\lambda L}-e^{-\lambda L}}{e^{\lambda L}+e^{-\lambda L}}
$$

where $v_{0}$ is the initial permeate flux at $x=0$, and ulim is the limiting exit axial velocity of the fiber. The initial permeate flux and the limiting exit axial velocity are, respectively, given by

$$
v_{0}=\frac{\Delta \mathrm{P}}{i R_{m}}
$$

and

$$
u_{\text {lim }}=\frac{\Delta \mathrm{P} \sqrt{r}}{2 i \sqrt{R_{m}}}
$$

Eq. (21) explicitly shows the impacts of various parameters on system performance. It can be directly used to analyze system performance in many cases. Eq. (23) shows that the maximum possible exit velocity is proportional to the transmembrane pressure and the square root of fiber inner radius, while inversely proportional to the water viscosity and the square root of membrane resistance.

\section{Simulations and Discussions}

In this section, the performance of the hollow fiber membrane system was simulated for various conditions. Unless other stated, the parameter values in Table 1 were used in the subsequent simulations. All the simulations were obtained with the numerical procedure. As a comparison and double check, the analytical solutions were also tried in some cases and almost exactly the same results were obtained. The choice of numerical procedure in the simulations was mainly for its easy expansion to the planned later study of the fouled fibers, for which the analytical solution will no longer be available.

\section{Frictional and kinetic pressure losses}

A numerical solution for the hollow fiber membrane system was presented in Figures $3 \mathrm{a}$ and $3 \mathrm{~b}$. The local transmembrane pressure was shown in Figure 3a with frictional and kinetic pressure losses. It can be seen that the kinetic pressure loss was very small that was practically zero in this case. The decrease on the transmembrane pressure was almost entirely caused by the frictional pressure loss. The corresponding local permeate velocity and the axial velocity were presented in Figure 3b. Similar to the transmembrane pressure, the local permeate velocity decreased from the open-end to the dead-end of the fiber. The average axial velocity always started from zero at the dead-end and increased to the maximum value at the open-end of the fiber.

Membrane resistance is reasonably anticipated to decrease with the advance in membrane materials and fabrication technology. It is of great interest to know how the decreased membrane resistance affects the frictional and kinetic pressure losses in the hollow fibers. The local transmembrane pressure and the frictional and kinetic pressure losses

\begin{tabular}{|c|c|c|}
\hline Parameter & Unit & Value \\
\hline Inner Radius & $\mathrm{m}$ & $0.2 \times 10^{-3}$ \\
\hline Fiber length & $\mathrm{m}$ & 3.0 \\
\hline Membrane resistance & $1 / \mathrm{m}$ & $1.12 \times 10^{12}$ \\
\hline Waterviscosity & $\mathrm{Pa} \cdot \mathrm{S}$ & $0.89 \times 10^{-3}$ \\
\hline Pressure & $\mathrm{Pa}$ & $0.5 \times 10^{5}$ \\
\hline
\end{tabular}

Table 1: Default parameter values used in numerical simulations. 

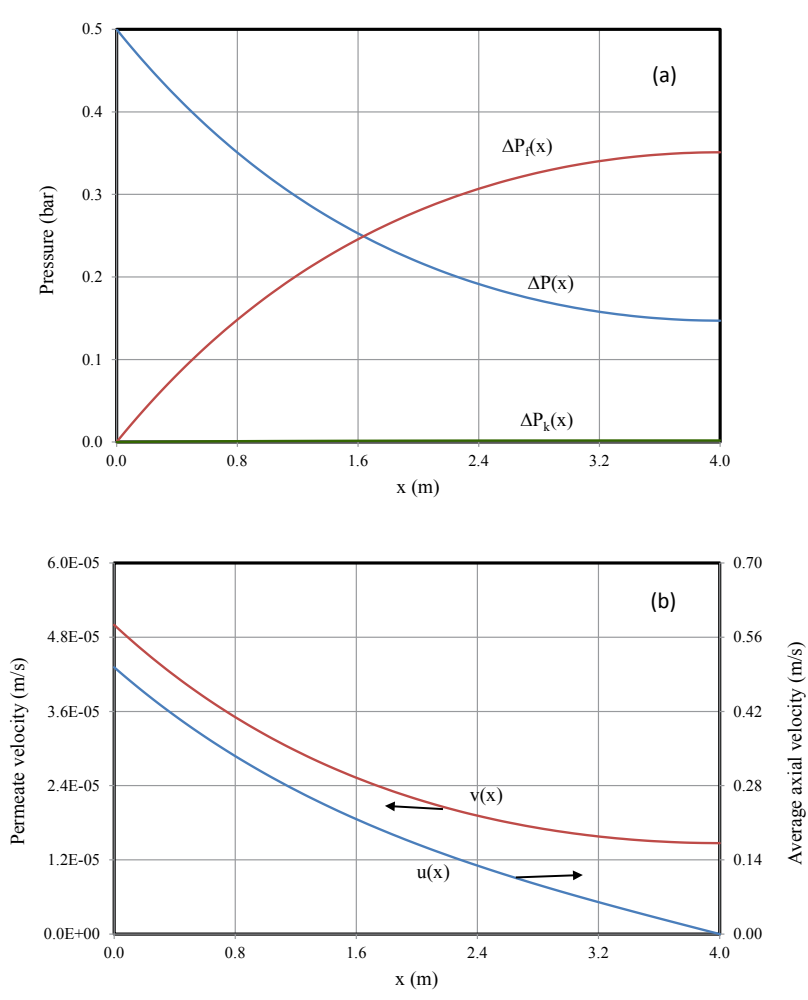

Figure 3: A numerical solution of the hollow fiber model with typical parameter values. (a) The transmembrane pressure along the fiber with frictional and kinetic pressure losses, and (b) the permeate and axial velocities along the fiber. The radius $r=0.4 \mathrm{~mm}$ and initial transmembrane pressure of 0.5 bar were used in the calculation.

were simulated and shown in Figures $4 \mathrm{a}-4 \mathrm{c}$ for membrane resistance of $1.12 \times 10111 / \mathrm{m}, 5.62 \times 10101 / \mathrm{m}$, and $1.12 \times 10101 / \mathrm{m}$, respectively. It can be seen that the kinetic pressure loss is no longer negligible for the lower membrane resistances. Figure $4 \mathrm{c}$ shows that the frictional and kinetic pressure losses are comparable in magnitude when the membrane resistance is $1.12 \times 10101 / \mathrm{m}$. For all the three membrane resistances used in the simulations, the kinetic pressure losses have to be considered for the sake of higher accuracy.

\section{Performance of hollow fiber filtration system}

For given membrane materials and fabrication technology of hollow fibers, the fiber length and radius are two important parameters of hollow fibers. The knowledge of their impacts on system performance is of paramount importance to the design of membrane system. In this section, the impacts of the fiber length and radius on the system performance (as represented by axial exit velocity) were studied under various conditions.

The impact of fiber length on the exit velocity for different membrane resistances was presented in the Figure 5. The resistance decreases with the increasing number on the graph. It can be seen that the exit velocity generally increases with fiber length. However, the increasing rate decreases as fiber length increases. The curves 1 to 3 in the figure represent the cases for the membrane resistance range of current hollow fibers. Curves 4 and 5 in the figure were produced with the membrane resistances of one third or one fifth of the typical resistance for current fibers as used to produce curve 2 . It can be seen from curves 4 and 5 that
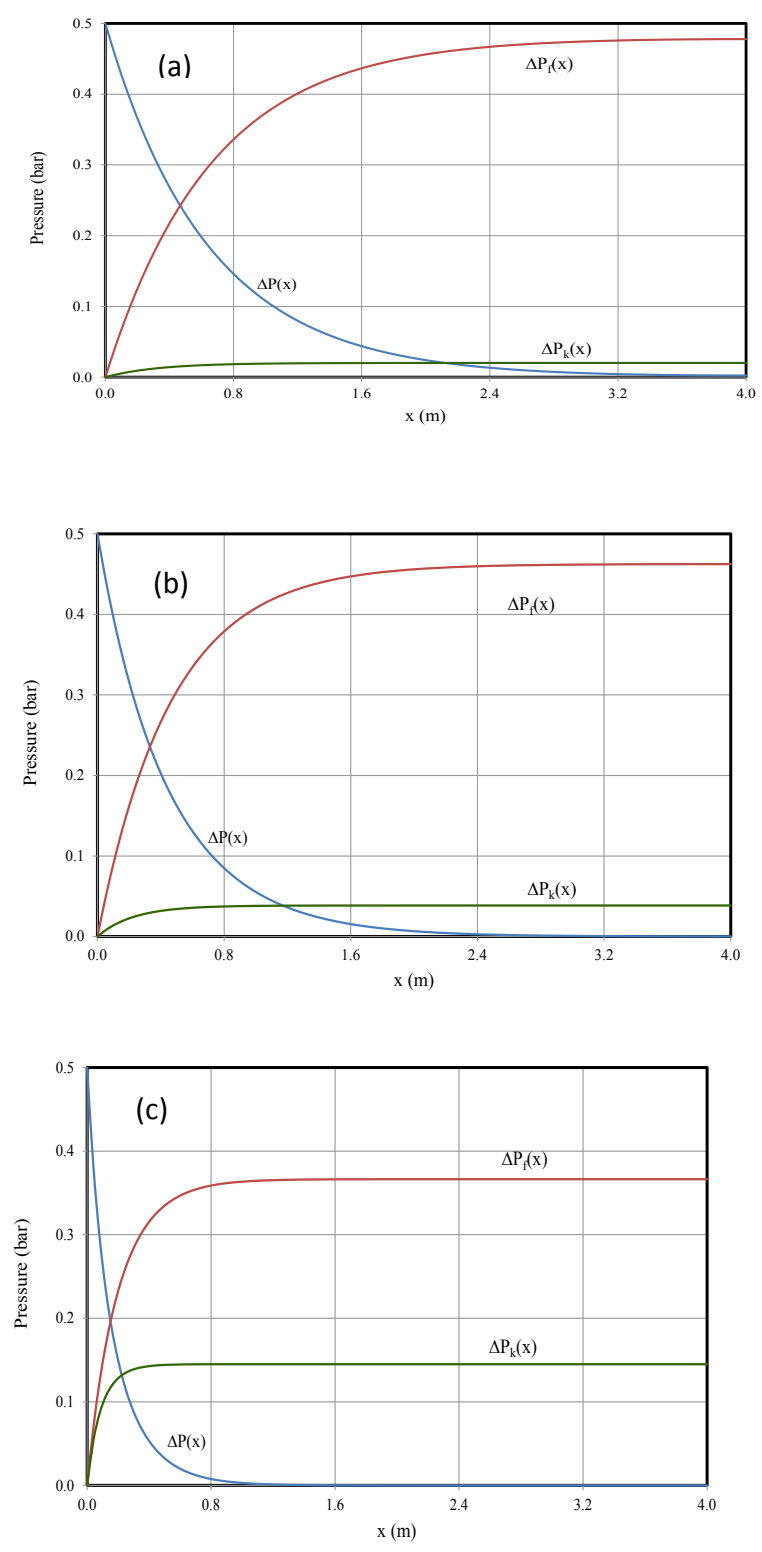

Figure 4: Numerical solutions of frictional and kinetic pressure losses for future low membrane resistances. (a) $R_{m}=1.12 \times 10^{11} 1 / \mathrm{m}$, (b) $R_{m}=5.62 \times 10^{10} 1 / \mathrm{m}$, and (c) $R_{m}=1.12 \times 10^{10} 1 / \mathrm{m}$. The radius $r=0.4 \mathrm{~mm}$ and initial transmembrane pressure of 0.5 bar were used in the calculation.

the exit velocities of the fiber no longer increase with fiber length when the length exceeds certain values. The limiting exit velocities calculated with Eq. (23) were presented in Table 2. The analytical solution can produce exactly the same exit velocities for all the membrane resistances. However, the numerical simulations showed the value of fiber length at which the limiting exit velocities could be obtained. Similar impact of fiber length on the exit velocity of the fiber can be seen for different initial transmembrane pressures as presented in Figure 6. The numbers on the curves indicated increasing transmembrane pressure used in the simulations. For all the transmembrane pressures simulated, the exit velocity keeps increasing with the fiber length.

Figure 7 presented profiles of the exit velocity as functions of fiber 
Citation: Liang S, Zhang H, Zhao Y, Song L (2016) Performance Modeling and Analysis of a Hollow Fiber Membrane System. J Membra Sci Technol 6: 144. doi:10.4172/2155-9589.1000144

Page 6 of 8

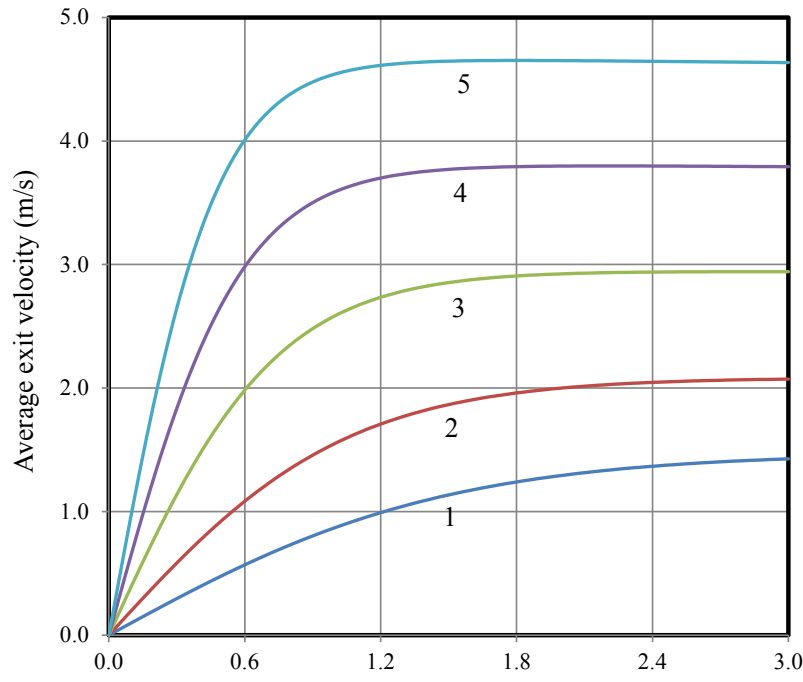

Figure 5: Axial velocity as a function of fiber length for various membrane resistances: 1) $\left.\left.\left.2.25 \times 10^{12} 1 / \mathrm{m}, 2\right) 1.12 \times 10^{12} / \mathrm{m}, 3\right) 5.62 \times 10^{11} 1 / \mathrm{m}, 4\right) 3.37$ $\times 10^{11} 1 / \mathrm{m}$, and 5) $2.25 \times 10^{11} 1 / \mathrm{m}$. Other parameters used in simulations are given in Table 1.

\begin{tabular}{|c|c|c|}
\hline Resistance , $\mathbf{1 / m}$ & Limiting velocity, $\mathbf{~ m} / \mathbf{s}$ & Note \\
\hline $2.25 \times 10^{12}$ & 1.48 & Curve 1 in Figure 5 \\
\hline $1.12 \times 10^{12}$ & 2.10 & Curve 2 in Figure 5 \\
\hline $5.62 \times 10^{11}$ & 2.96 & Curve 3 in Figure 5 \\
\hline $3.37 \times 10^{11}$ & 3.82 & Curve 4 in Figure 5 \\
\hline $2.25 \times 10^{11}$ & 4.68 & Curve 5 in Figure 5 \\
\hline
\end{tabular}

Table 2: Limiting exit velocity of hollow fiber.

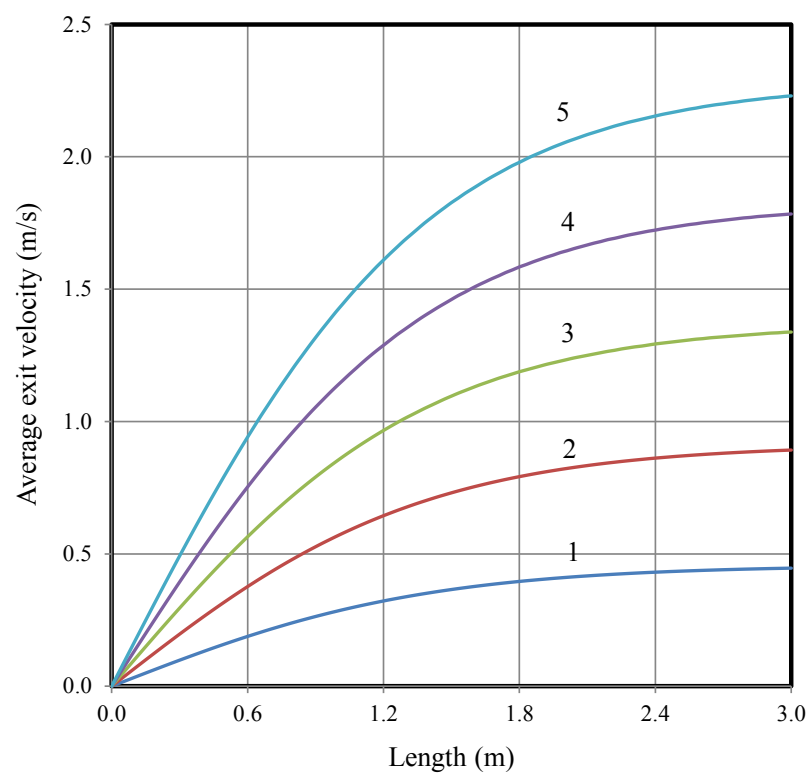

Figure 6: Axial velocity as a function of fiber length for various initial transmembrane pressures: 1) 0.5 bar, 2) 1.0 bar, 3) 1.5 bar, 4) 2.0 bar, and 5) 2.5 bar. Other parameters used in simulations are given in Table 1.

length for different fiber radii. Comparing to resistance and pressure, the radius of hollow fiber is by far a much more influential parameter on the performance of the fiber membrane. The numbers on the curves in the figure indicate simulation results for increasing fiber radius. Instead of monotonic trend as for resistance and pressure, the impact of fiber radius on performance is more complicated and interesting. The hollow fiber of smaller radius produces more permeates (higher exit velocity) for shorter fiber but reaches a plateau value sooner as fiber length increases. It can be seen on Figure 7 that the hollow fiber with the radius of $0.15 \mathrm{~mm}$ (curve 1) produces the most permeate for fiber length up to $0.85 \mathrm{~m}$. However, the maximum axial velocity (ca. $1.6 \mathrm{~m} / \mathrm{s}$ )

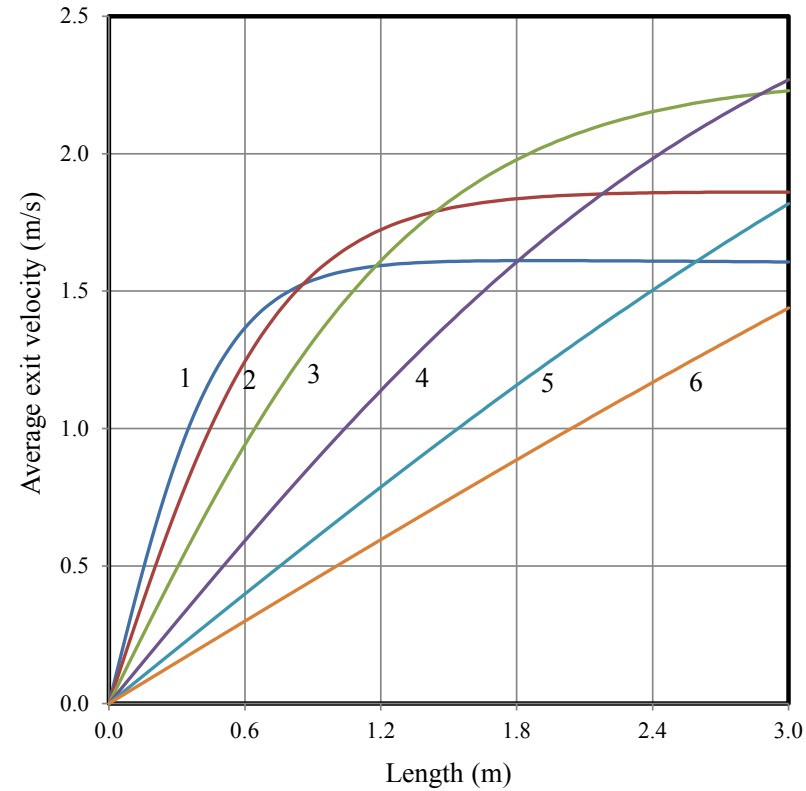

Figure 7: Axial velocity as a function of fiber length for various fiber radiuses: 1) $0.15 \mathrm{~mm}$, 2) $0.20 \mathrm{~mm}$, 3) $0.30 \mathrm{~mm}$, 4) $0.50 \mathrm{~mm}$, 5) $0.75 \mathrm{~mm}$, and 6) $1.0 \mathrm{~mm}$ Other parameters used in simulations are given in Table 1.

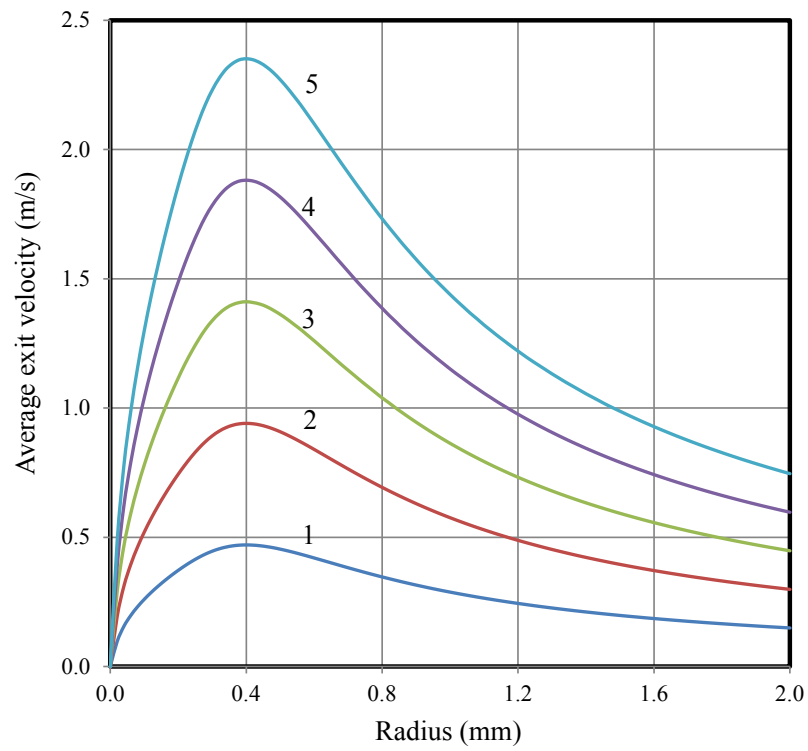

Figure 8: Axial velocity as a function of fiber radius for various initial transmembrane pressures: 1) 0.5 bar, 2) 1.0 bar, 3) 1.5 bar, 4) 2.0 bar, and 5) 2.5 bar. Other parameters used in simulations are given in Table 1. 


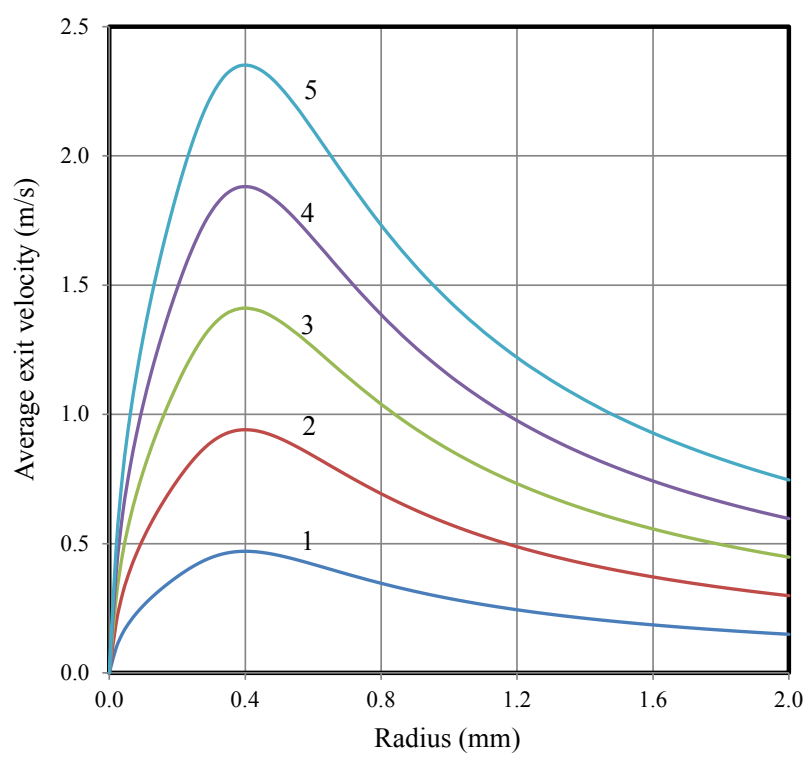

Figure 9: Axial velocity as a function of fiber radius for various membrane resistances: 1) $\left.\left.\left.2.25 \times 10^{12} 1 / \mathrm{m}, 2\right) 1.12 \times 10^{12} / \mathrm{m}, 3\right) 5.62 \times 10^{11} 1 / \mathrm{m}, 4\right) 3.37$ $\times 10^{11} 1 / \mathrm{m}$, and 5) $2.25 \times 10^{11} 1 / \mathrm{m}$. Other parameters used in simulations are given in Table 1.

for the fiber is reached at the length of $1.8 \mathrm{~m}$. The fiber with radius of $0.20 \mathrm{~mm}$ (curve 2) produces the most permeate for the fiber length between about $0.85 \mathrm{~m}$ to $1.40 \mathrm{~m}$, where it is overtaken by the fiber of radius $0.30 \mathrm{~mm}$ (curve 3). Curves 5 and 6 represented radius of 0.50 $\mathrm{mm}$ and $1.0 \mathrm{~mm}$ and the radii are too large for the conditions used in the simulations. They cannot produce the highest exit velocity in the whole range of fiber length simulated.

The impact of fiber radius on exit velocities was presented in Figures 8 and 9 for groups of pressures and resistances, respectively. It can be seen that there is a peak value of exit velocity at certain radius for each curve, which do not appear in the previous figures. Figure 8 shows that transmembrane pressure does not affect the radius value at which the peak exit velocity occurs although the peak velocity is strongly affected by pressure. Figure 9, on the contrary, shows that the decrease in the membrane resistance postpones the occurrence of peak exit velocity to a larger radius. It suggests that fibers with larger radius should be made to accommodate the membranes with lower resistance. It is possible to mathematically find the optimal radius for given sets of parameters by taking derivative of Eq. (13) and setting it to zero. However, the simulations of the performance of the hollow fiber as a function of radius can provide a direct graphical and vivid presentation of the result that cannot be matched by the single value from mathematical determination.

\section{Conclusions}

A model for the performance of a hollow fiber membrane system was developed by rigorously considering both frictional and kinetic pressure losses along the fiber. By numerical simulations of the complete model, it was demonstrated that the kinetic pressure loss is much smaller than the frictional pressure loss for the current hollow fiber membranes. Therefore, the performance of the hollow fiber membrane systems can be reasonably modeled by considering frictional pressure loss only. The analytical solution for the reduced model was derived with the driving pressure as the primary control parameter. Simulations showed that there exists a maximum exit axial velocity (correspondingly a maximum average permeate flux) with respect to driving pressure for given set of parameters. For given membrane materials (resistance) and fiber length, an optimal radius of the fiber can be determined to maximize axial velocity. The interesting simulations findings cannot be done with the model derived based on average permeate flux as a control parameter.

\section{References}

1. Baker RW (2004) Membrane Technology and Applications. (2ndedn), John Wiley \& Sons Ltd., Chichester.

2. Carroll $T$ (2001) The effect of cake and fibre properties on flux declines in hollow-fibre microfiltration membranes. J Membr Sci 189: 167-178.

3. Yeh HM (2009) Exponential model analysis of permeate flux for ultrafiltration in hollow-fiber modules by momentum balance. Chem Eng J 147: 202-209.

4. Keller AA, Bierwagen BG (2001) Hydrophobic hollow fiber membranes for treating MTBE-contaminated water. Environ Sci Technol 35: 1875-1879.

5. Guo H, Wyart Y, Perot J, Nauleau F, Moulin P (2010) Low-pressure membrane integrity tests for drinking water treatment: A review. Water Res 44: 41- 57

6. Sun SP, Hatton TA, Chung TS (2011) Hyperbranched polyethyleneimine induced cross-linking of polyamide-imide nanofiltration hollow fiber membranes for effective removal of ciprofloxacin. Environ Sci Technol 45: 4003-4009.

7. Xiao P, Xiao F, Wang DS, Qin T, He SP (2012) Investigation of organic foulants behavior on hollow-fiber UF membranes in a drinking water treatment plant Sep Purif Technol 95: 109-117.

8. Liu TY, Zhang RX, Li Q, Van der Bruggen B, Wang XL (2014) Fabrication of a novel dual-layer (PES/PVDF) hollow fiber ultrafiltration membrane for wastewater treatment. J Membr Sci 472: 119-132.

9. Aziz CE, Fitch MW, Linquist LK, Pressman JG, Georgiou G, et al. (1995) Methanotrophic biodegradation of trichloroethylene in a hollow-fiber membrane bioreactor. Environ Sci Technol 29: 2574-2583

10. Fenu A, De Wilde W, Gaertner M, Weemaesde M, Gueldre G, et al. (2012) Elaborating the membrane life concept in a full scale hollow-fibers MBR. J Membr Sci 421: 349-354.

11. Robles A, Ruano MV, Ribes J, Ferrer J (2013) Factors that affect the permeability of commercial hollow-fibre membranes in a submerged anaerobic MBR (HF-SAnMBR) system. Water Res 47: 1277-1288.

12. Chellam S, Jacangelo JG, Bonacquisti TP (1998) Modeling and experimental verification of pilot-scale hollow fiber, direct flow microfiltration with periodic backwashing. Environ Sci Technol 32: 75-81.

13. Kim AS, Lee YT (2011) Laminar flow with injection through a long dead-end cylindrical porous tube: Application to a hollow fiber membrane. AIChE J 57 : 1997-2006.

14. Serra C, Clifton MJ, Moulin P, Rouch JC, Aptel P (1998) Dead-end ultrafiltration in hollow fiber modules: Module design and process simulation. J Membr Sci 145: $159-172$

15. Yoon SH, Lee S, Yeom IT (2008) Experimental verification of pressure drop models in hollow fiber membrane. J Membr Sci 310: 7-12.

16. Li X, Li J, Wang J, Wang H, He B, et al. (2014) Experimental investigation of local flux distribution and fouling behavior in double-end and dead-end submerged hollow fiber membrane modules. J Membr Sci 453: 18-26.

17. Ghidossi R, Daurelle J V, Veyret D, Moulin P (2006) Simplified CFD approach of a hollow fiber ultrafiltration system. Chem Eng J 123: 117-125.

18. Ghidossi R, Veyret D, Moulin P (2006) Computational fluid dynamics applied to membranes: State of the art and opportunities. Chem Eng Process 45: 437-454

19. Chang S, Fane AG (2001) The effect of fibre diameter on filtration and flux distribution - relevance to submerged hollow fibre modules. J Membr Sci 184 221-231. 
Citation: Liang S, Zhang H, Zhao Y, Song L (2016) Performance Modeling and Analysis of a Hollow Fiber Membrane System. J Membra Sci Technol 6: 144. doi:10.4172/2155-9589.1000144

Page 8 of 8

20. Chang S, Fane AG, Vigneswaran S (2002) Modeling and optimizing submerged hollow fiber membrane modules. AIChE J 48: 2203-2212.

21. Chang S, Fane AG, Waite TD (2006) Analysis of constant permeate flow filtration using dead-end hollow fiber membranes. J Membr Sci 268: 132-141.

22. Lee S, Park PK, Kim JH, Yeon KM, Lee CH (2008) Analysis of filtration characteristics in submerged microfiltration for drinking water treatment. Water Res 42: 3109-3121.

23. Liu L, Ding Z, Lu Y, Ma R (2010) Modeling the bubbling enhanced microfiltration for submerged hollow fiber membrane module. Desalination 256: 77-83.

24. Yoon SH, Kim HS, Yeom IT (2004) Optimization model of submerged hollow fiber membrane modules. J Membr Sci 234: 147-156.

25. Lee HM, Lee CH, Chung KY, Lee S (2004) Prediction model for transmembrane pressure in a submerged hollow-fiber microfiltration membrane. Separ Sci Technol 39: 1833-1856.

26. Azarbadegan A, Eames I, Sharma S, Cass A (2011) Computational study of parallel valveless micropumps. Sensor Actuator B 158: 432-440.

27. Romeo E, Royo C, Monzón A (2002) Improved explicit equations for estimation of the friction factor in rough and smooth pipes. Chem Eng J 86: 369-374.

28. Chen NH (1979) An explicit equation for friction factor in pipe. Ind Eng Chem Fundam 18: 296-297. 